\title{
Study of the possibility of reducing the angular errors of circular optical scales fabricated by lift-off photolithography
}

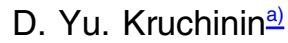 \\ First President of Russia B. N. Yeltsin Ural Federal University, Ekaterinburg, Russia
}

(Submitted December 5, 2012)

Opticheskǐ̌ Zhurnal 80, 42-45 (August 2013)

\begin{abstract}
This paper discusses the possibilities of reducing the angular errors of circular optical scales fabricated by lift-off photolithography, using photomasks synthesized by means of a CLWS-300 laser image generator. (C) 2013 Optical Society of America.
\end{abstract}

OCIS code: (110.5220) Photolithography.

http://dx.doi.org/10.1364/JOT.80.000499

\section{INTRODUCTION}

The method of lift-off photolithography has been widely used in fabricating precision circular optical scales (dials). . $-2^{-}$ The most important technical characteristic of dials is the angular error of placement of the rulings, and the most often encountered form of angular error is the angular error of placement of the diameters of the axes of the rulings. For the indicated form of angular error, the result of a measurement on the AS700 apparatus can be represented as 3,4

$$
F(x)=F_{\text {mon }}(x)+F_{\text {pm }}(x)+F_{\exp 2}(x)+F_{\text {sub }}(x),
$$

where $x$ is the angular position of the ruling, $F_{\text {mon }}(x)$ are the errors introduced by the AS700 monitoring apparatus, $F_{\mathrm{pm}}(x)$ are the errors introduced by the CLWS-300 laser image generator ${ }^{5}$ via the topology of the photomask, $F_{\exp 2}(x)$ are the errors introduced by the exposure operation because the exposure-setting plate deviates from a plane and forms a gap of variable size between the substrate and the photomask, and $F_{\text {sub }}(x)$ are the errors caused by the shape of the substrate (wedge, deviation of the working side from a plane, etc.).

The phase-statistical method makes it possible to separate $F_{\text {mon }}(x)$ from $F_{\text {pm }}(x)+F_{\exp 2}(x)+F_{\text {sub }}(x)$. - The dependences of the angular error of placement of the diameters of the axes of the dial rulings will then have the form

$$
F_{\text {dial }}(x)=F_{\text {pm }}(x)+F_{\exp 2}(x)+F_{\text {sub }}(x) .
$$

The $F_{\exp 2}(x)$ dependences of the angular error of placement of the diameters of the axes of the dial rulings introduced by the exposure operation are mainly determined by the second harmonic. - It can be assumed that these dependences are fairly constant for a specific exposure setting. At the same time, the indicated dependences can change as a result of a change of the amplitude of the second harmonic when the tilt angle of the exposure-setting plate changes.- The $F_{\mathrm{pm}}(x)$ dependences of the angular error of placement of the diameters of the axes of the dial rulings introduced by the CLWS-300 laser image generator via the photomask topology are associated with the corresponding $F_{\text {im }}(x)$ dependences of the circular optical scales fabricated by means of the CLWS-300 image generator,- following the relationship

$$
F_{\text {im }}(x)=-F_{\mathrm{pm}}(-x) \text {. }
$$

It has been established that the dependences of the angular errors of placement of the diameters of the axes of the rulings of circular optical scales fabricated by means of the CLWS300 laser image generator are determined by the specific synthesis conditions and can significantly differ from each other.? These dependences are mainly determined by the second as well as the fourth harmonic. The accumulated errors of placement of the diameters of the axes of the rulings can vary from 1.5 to 4 or more arc seconds. The dependences of the angular errors of placement of the diameters of the axes of the rulings of circular optical scales fabricated by means of a CLWS-300 laser image generator are thus distinguished by the set of harmonics (their amplitudes and phases), as well as by the size of the accumulated errors. Further studies showed that, when circular optical scales are synthesized on free-lying substrates, the dependences of the angular errors of placement of the diameters of the axes of the rulings are determined by the second harmonics, which are close in phase and amplitude, while the accumulated errors do not exceed 1.5 arc sec. - This means that there is a possibility of obtaining photomasks of circular optical scales the dependences of the angular errors of which are predetermined to a sufficient degree.

The $F_{\text {sub }}(x)$ dependences of the angular error of the placement of the diameters of the axes of the dial rulings caused by the shape of the substrate are specific to each substrate but are apparently largely determined by the second harmonic.? There are significant difficulties in separating the $F_{\text {sub }}(x)$ dependences from the other dependences. The accumulated error $F_{\text {sub }}(x)$ of placement of the axes of the rulings caused by the shape of the substrate is about 0.7 arc sec for a specific substrate. ? It was established earlier- that, when dials are fabricated by lift-off photolithography, the angular errors can be either increased or decreased. The conditions under which the accumulated angular errors increase or decrease were not determined. The cause of the increase or decrease of the 
accumulated angular errors is apparently the interaction of the $F_{\mathrm{pm}}(x)$ and the $F_{\text {sub }}(x)+F_{\text {exp } 2}(x)$ dependences. The accumulated angular errors of the dials will increase if the phases of the second harmonics of these dependences are close, but the accumulated angular errors will decrease if the second harmonics are out of phase. The resulting photomasks, which have definite and repeating dependences of the angular errors, give the possibility of creating a technology for obtaining dials that have the minimum angular errors. The task of this investigation is to determine whether the angular errors of circular optical scales (dials) fabricated by lift-off photolithography can be reduced because of the interaction of the $F_{\mathrm{pm}}(x)$ and $F_{\text {sub }}(x)+F_{\exp 2}(x)$ dependences.

\section{EXPERIMENTAL TECHNIQUE}

A CLWS-300 laser image generator was used to fabricate a photomask that consists of a closed circular scale consisting of 360 transparent rulings $8 \mu \mathrm{m}$ wide on a scale $90 \mathrm{~mm}$ in diameter. The zero ruling is narrowed on each side by $0.5 \mu \mathrm{m}$. The photomask was recorded on a free-lying chrome-plated substrate.

Three polished substrates having a deviation from a plane no worse than $N=10$ and $\Delta N=2$ were used for the investigation. A scale was formed on each substrate by multiple lift-off photolithography, using a chromium masking coating. $1.2-$ The zero ruling for each formation of the scale was at approximately the same site relative to the exposure-setting plate. The scale was formed with the photomask placed on the exposuresetting plate in two positions, the angle between which was $90^{\circ}$. For the first position of the photomask, a scale was formed on each substrate with a slope angle of the exposure-setting plate equal to $0^{\circ}$. For the second position of the photomask, a scale was formed on each substrate with slope angles of the exposuresetting plate equal to $1^{\circ}, 0^{\circ}$, and $-0.5^{\circ}$. Eighteen measurements were made on the AS700 dial-testing apparatus for each scale. The measurements were made with the dial rotated relative to the spindle axis of the apparatus by $20^{\circ}$. The position of the narrowed ruling was determined from the characteristic isolated overshoot on the dependences of the angular error of placement of the boundaries of the rulings. Then, in accordance with the phase-statistical method, all the resulting dependences of the angular errors of placement of the diameters of the axes of the rulings were averaged, with the narrowed ruling being taken each time for the zero ruling. This gave the $F_{\text {dial }}(x)$ dependence for each scale. After each series of measurements, the scale was removed chemically and was then deposited afresh.

\section{EXPERIMENTAL RESULTS AND DISCUSSION}

The dependences of the angular errors of placement of the diameters of the axes of the rulings corresponding to Eq. (2) are obtained for each substrate from the experimental results. The accumulated errors of placement of the diameters of the axes of the rulings for the dials fabricated with the first position of the photomask and a slope angle of the exposure-setting plate equal to $0^{\circ}$ have values from 2.41 to $2.49 \mathrm{arc} \mathrm{sec}$, and this exceeds the corresponding accumulated error of the circular scale of the photomask $(1.5 \mathrm{arc} \mathrm{sec})$. $^{\frac{8}{-}}$ The subsequent studies were therefore carried out for dials fabricated with the second position
TABLE 1. Accumulated angular errors of placement of the diameters of the axes of the rulings

\begin{tabular}{lcccc}
\hline \hline & \multicolumn{3}{c}{ Accumulated Angular Error (arc sec) } \\
\cline { 3 - 5 } Position of & $\begin{array}{c}\text { Slope Angle of } \\
\text { Photomask }\end{array}$ & $\begin{array}{c}\text { Substrate } \\
\text { To. } 1\end{array}$ & $\begin{array}{c}\text { Substrate } \\
\text { No. 2 }\end{array}$ & $\begin{array}{c}\text { Substrate } \\
\text { No. 3 }\end{array}$ \\
\hline 1 & 0 & 2.41 & 2.49 & 2.41 \\
2 & 0 & 1.33 & 2.01 & 1.25 \\
2 & 1 & 1.91 & 2.08 & 1.17 \\
2 & -0.5 & 1.04 & 1.23 & 0.95 \\
\hline \hline
\end{tabular}

of the photomask on the exposure-setting plate. The phase of the $F_{\mathrm{pm}}(x)$ dependence in this case is displaced by $90^{\circ}$, while the phase of the second harmonic of the indicated dependence changes so that it is out of phase. As a result, the accumulated angular errors of placement of the diameters of the axes of the rulings for a slope angle of the exposure-setting plate equal to $0^{\circ}$ range from 1.25 to $2.01 \mathrm{arc} \mathrm{sec}$. Changing the slope angle of the exposure-setting plate can change the accumulated error of placement of the diameters of the axes of the rulings. An increase of the slope angle of the exposure-setting plate by $1^{\circ}$ results, on the whole, in some increase of the accumulated error. A decrease of the slope angle of the exposure-setting plate by $0.5^{\circ}$ results in an appreciable reduction of the values of the accumulated errors. (The results of this part of the paper are collected in Table 1.) It is impossible to reduce the slope angle of the exposure-setting plate further because of its design features. The $F_{\text {dial }}(x)$ dependence of the angular error of placement of the diameters of the axes of the rulings for the scale deposited on substrate no. 3 with a slope angle of the exposure-setting plate equal to $-0.5^{\circ}$ is shown in Fig. 1 .

The interaction of the $F_{\mathrm{pm}}(x)$ and $F_{\text {sub }}(x)+F_{\text {exp } 2}(x)$ dependences can cause the accumulated errors to either increase or decrease. The stability of the $F_{\mathrm{pm}}(x)$ and $F_{\exp 2}(x)$ dependences makes it possible to determine fairly accurately the exposure conditions (the placement of the photomask on the exposure-setting plate) for which the angular errors are minimized. The interaction of the $F_{\mathrm{pm}}(x)$ and $F_{\text {sub }}(x)+F_{\exp 2}(x)$ dependences under definite conditions makes it possible to reduce the accumulated angular errors of placement of the diameters of the axes of the rulings to values close to 1 arc sec. The scatter of the values of the accumulated angular errors of placement of the diameters of the axes of the rulings for

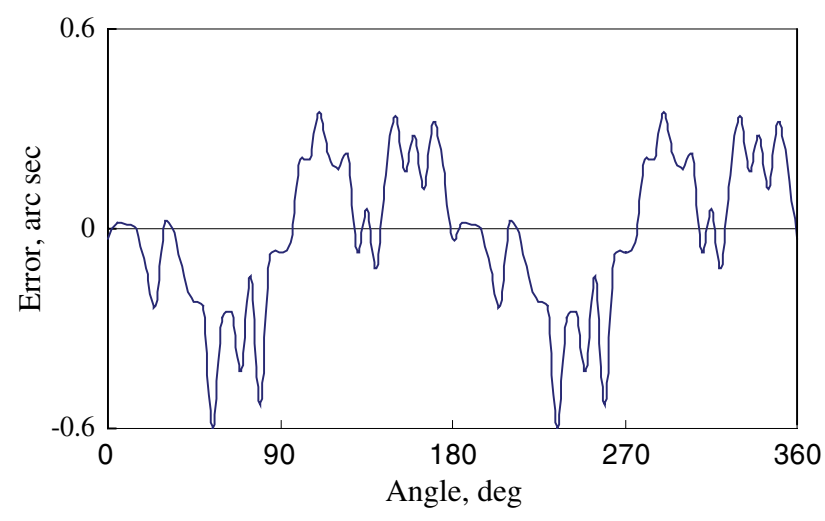

FIG. 1. The $F_{\text {dial }}(x)$ dependence of the angular errors of placement of the diameters of the axes of the rulings. Substrate No. 3, slope angle $-0.5^{\circ}$. 
different substrates under other identical conditions is caused by the individual character of the $F_{\text {sub }}(x)$ dependences.

\section{CONCLUSION}

Our studies have allowed us to determine that there is a possibility of reducing the accumulated angular error of placement of the diameters of the axes of the dial rulings to values close to 1 arc sec because of the interaction of the $F_{\mathrm{pm}}(x)$ dependence of the angular errors introduced by the CLWS-300 laser image generator via the topology of the photomask and the $F_{\exp 2}$ dependence of the angular errors introduced by the exposure operation plus the $F_{\text {sub }}(x)$ dependence influenced by the substrate shape. Apparently, if the $F_{\text {sub }}(x)$ dependence is determined and separated out for each substrate, the possibility appears of compensating the $F_{\mathrm{pm}}(x)$ and $F_{\exp 2}(x)$ dependences more fully. The conditions for carrying out the exposure can change in this case. The accumulated errors can be reduced by displacing the phase of the $F_{\mathrm{pm}}(x)$ dependence when the photomask is synthesized, in order to more accurately approximate the phase shift to $90^{\circ}$ relative to the phase of the $F_{\exp 2}(x)$ dependence.

${ }^{a)}$ Email: klip3@mail.ru

${ }^{1}$ D. Yu. Kruchinin, "Method of fabricating optical scales by lift-off photolithography,” Russian Patent No. 2370799 (2009).
${ }^{2}$ D. Yu. Kruchinin, "Photolithography in the production of circular optical scales at the Ural Optomechanical Factory," Opt. Zh. 75, No. 4, 92 (2008) [J. Opt. Technol. 75, 282 (2008)].

${ }^{3}$ D. Yu. Kruchinin, O. V. Anisimova, and A. S. Tyryshkina, "Study of the angular errors of dials fabricated by reverse photolithography," Opt. Zh. 76, No. 6, 70 (2009) [J. Opt. Technol. 76, 373 (2009)].

${ }^{4} \mathrm{D}$. Yu. Kruchinin, "Study of how the angles of incidence of the rays of actinic radiation on the surface of a photomask affect the angular errors of dials fabricated by lift-off photolithography," Opt. Zh. 77, No. 6, 46 (2010) [J. Opt. Technol. 77, 387 (2010)].

${ }^{5}$ Yu. F. Abramov, V. P. Kir'yanov, A. V. Kir'yanov, S. A. Kokarev, D. Yu. Kruchinin, Yu. V. Chuguĭ, and O. B. Yakovlev, "Modernizing the optical divider production of the Ural Optomechanical Factory on the basis of up-to-date laser-computer and photolithographic technologies," Opt. Zh. 73, No. 8, 61 (2006) [J. Opt. Technol. 73, 544 (2006)]

${ }^{6}$ S. A. Bartik, S. E. Frizin, V. P. Kiryanov, A. V. Kiryanov, S. A. Kokarev, D. Y. Kruchinin, V. G. Nikitin, and O. B. Yakovlev, "Development of a technique for the determination of metrological parameters of technological system CLWS-300/C for synthesis of high precision angular measuring structures," in Tenth IMEKO TC7 International Symposium, St. Petersburg, 2004, pp. 316-320

${ }^{7}$ D. Yu. Kruchinin and O. B. Yakovlev, "Study of the angular errors of circular optical scales fabricated by means of the CLWS-300 laser image generator," Opt. Zh. 78, No. 6, 47 (2011) [J. Opt. Technol. 78, 383 (2011)].

${ }^{8}$ D. Yu. Kruchinin, O. B. Yakovlev, and M. P. Andronov, "Study of how the synthesis conditions of circular optical scales fabricated using a CLWS-300 laser image generator affect their angular errors," Opt. Zh. 79, No. 7, 41 (2012) [J. Opt. Technol. 79, 412 (2012)].

${ }^{9}$ D. Yu. Kruchinin and O. B. Yakovlev, "Photomasks for the production of optical scales," in Transactions of the International Conference on Applied Optics-2006, St. Petersburg, 2006, vol. 2, pp. 111-114. 